\title{
The potential of adipose-derived stem cell subpopulations in regenerative medicine
}

\author{
Richard JP Smith ${ }^{1}$ \& Adam J Reid*,1,2 \\ ${ }^{1}$ Blond Mclndoe Laboratories, Division of Cell Matrix Biology \& Regenerative Medicine, School of Biological Sciences, Faculty of \\ Biology Medicine \& Health, University of Manchester, Manchester Academic Health Science Centre, Manchester M13 9PL, UK \\ ${ }^{2}$ Department of Plastic Surgery \& Burns, Wythenshawe Hospital, Manchester University NHS Foundation Trust, Manchester \\ Academic Health Science Centre, Manchester, UK \\ *Author for correspondence: adam.reid@manchester.ac.uk
}

"The growing awareness of the importance and utility of stem cells has so far gone hand in hand with advancements in regenerative medicine. At the same time, the complexity of the therapeutic potential of stem cells heralds the need for more targeted and patient-specific treatments."

First draft submitted: 17 March 2018; Accepted for publication: 15 May 2018; Published online:

6 July 2018

Keywords: adipose-derived stem cells $\bullet$ autologous surgery $\bullet$ CD271 $\bullet$ CD34 $\bullet$ CD90 • fat grafting $\bullet$ personalized medicine • stem cell enrichment • stromal vascular fraction • subpopulation selection

As our understanding of cellular biology advances, so must our application of this knowledge. The clinical approaches to problems of regenerative medicine must inevitably become more reliant on our understanding of cellular mechanisms and interactions.

The growing awareness of the importance and utility of stem cells has so far gone hand in hand with advancements in regenerative medicine. At the same time, the complexity of the therapeutic potential of stem cells heralds the need for more targeted and patient-specific treatments.

The past few decades have seen an increase in our understanding of distinct subpopulations of mesenchymal stem cells. An area that has seen much interest is that of adipose-derived stem cell (ASC) subpopulations, due to their translational potential and abundant source material.

There is some evidence of the therapeutic benefit of subpopulations of ASCs compared with stromal vascular fraction (SVF). Derived from digested adipose tissue, SVF is a heterogeneous population of cells that is used in a number of regenerative procedures. Despite its abundance in the literature, very little standardization in SVF extraction and purification methods has resulted in uncertainty about its precise therapeutic value [1].

\section{The use of SVF/heterogeneous ASCs in tissue regeneration}

ASCs are capable of differentiating into chondrocytes, osteocytes and myocytes as well as adipocytes, and possess the ability to differentiate into more diverse lineages such as endothelial cells and neurons. As such, SVF (containing a heterogeneous ASC population) has been utilized in a wide range of applications.

SVF has been shown to enhance skeletal muscle regeneration in mice [2], and vocal fold regeneration in rats [3]. SVF injections could even improve pain relief in patients suffering from osteoarthritis [4]. SVF also holds potential in the regeneration of peripheral nerves, with SVF-enriched bioengineered conduits producing improved nerve repair in a rat sciatic nerve injury model [5].

Fat grafts enriched with SVF have been proven to exhibit improved survival compared with nonenriched grafts in several animal studies [6,7]. Human studies of SVF-enriched fat grafting have also shown promise, illustrating the safety and efficacy of SVF enrichment in enhancing volume retention $[8,9]$.

\section{The use of ASC subpopulations in tissue regeneration}

The SVF is a highly heterogeneous population containing cells that cannot be ideal therapeutic agents. Although studies utilizing SVF show promise, they often lack a comprehensive characterization of the ASC populations

Future Medicine 
present within their cell preparations, leaving us unsure of the ideal cellular composition of a regenerative SVF isolate.

ASCs have been shown to release a large number of factors and perform a variety of functions in adipose tissue. As such it is unlikely that one subtype of ASC is performing all these functions. Accordingly, distinct subpopulations of ASC with different phenotypes have been detected in vivo [10]. Since these subpopulations are performing different functions in vivo, a logical approach to tissue engineering is selecting subpopulations that are most beneficial to the required role. Therefore, the best approach is to investigate characteristics of various ASC subpopulations by identifying and selecting these phenotypes by unique complements of cell surface markers.

Considerable in vitro and in vivo data present several subpopulations as being highly angiogenic and adipogenic compared with the highly heterogeneous SVF population:

CD29 is a surface marker also known as integrin $\beta 1$, and is a marker of mesenchymal cells. Human CD29+ ASCs, after in vitro chondrogenic differentiation followed by 6 weeks of implantation in mice, show increased expression of chondrogenic markers compared with a $\mathrm{CD} 105^{+}$subpopulation [11].

ASCs expressing the surface marker CD34 have been studied extensively. CD34 is a surface glycoprotein involved in cell adhesion, and is typically used as a marker for hematopoietic cells. Analysis of Oil Red O and calcium content after induction toward adipocytes and osteocytes showed that human $\mathrm{CD} 34^{+}$ASCs exhibit increased adipogenic and osteogenic potential compared with the unsorted population, as well as an increased proliferative ability [12]. One in vivo study showed that the human $\mathrm{CD} 34^{+} / \mathrm{CD} 31^{-}$ASC population improves blood flow and capillary density in a mouse model of ischemia, compared with the CD34 /CD31- population [13].

CD90 is a glycoprotein mainly found in leukocytes. The general CD90 $0^{+}$population of human ASCs, sorted after $36 \mathrm{~h}$ in culture and differentiated for 14 days, exhibits higher alkaline phosphatase and alizarin red staining compared with other subpopulations (CD90- $\mathrm{CD} 105^{\mathrm{High}}$ and $\mathrm{CD} 105^{\mathrm{Low}}$ ), and higher expression of osteogenic genes, suggesting this population may have improved osteogenic capacity [14].

The $\mathrm{CD} 34^{+} / \mathrm{CD} 90^{+}$human ASC population shows much promise. Sorting for these double positive cells immediately after extraction produces a population capable of differentiating into endothelial cells that go on to form vascular structures in vitro, presenting $\mathrm{CD} 34^{+} / \mathrm{CD} 90^{+}$ASCs as a means of improving vascularization of tissues [15]. Cell cycle analysis on this population revealed a high rate of proliferation compared with $\mathrm{CD} 34^{-} / \mathrm{CD}^{-} 0^{-}$ ASCs, and the double positive populations formed spheres in culture, where the double negative population did not [15]. When the $\mathrm{CD} 34^{+} / \mathrm{CD} 90^{+}$population is seeded in collagen constructs and implanted in mice for 30 days, the formation of vascularized adipose tissue is seen, and this is not observed in collagen constructs seeded with unsorted ASCs [16]. Histology of the resulting tissue revealed adipose, connective and vascular tissue had been formed, and real time PCR showed the expression of adiponectin, PPARG, VEGF, fibronectin and collagen type 1 in this tissue formed from $\mathrm{CD} 34^{+} / \mathrm{CD} 90^{+}$ASCs [16].

Another promising subpopulation identifier for tissue engineering is CD271. Also known as low-affinity nerve growth factor receptor or p75, CD271 is a receptor found highly expressed in the glial cells of the central and peripheral nervous systems. ASCs expressing CD271 have been found to also express Notch1, Rex1 and Nestin, markers of self-renewal, pluripotency and cytoskeletal competency respectively [17-19].

Murine CD271+ ASCs can differentiate into multiple lineages in vitro, and the rate of differentiation into adipo-, chondro- and osteocytes (stained by Oil Red O, aggrecan and collagen type II, and Alizarin Red, respectively) was faster for $\mathrm{CD} 271^{+}$cells compared with CD271- cells [20]. Quirici et al. also demonstrated an increased rate of adipoand osteogenic differentiation in $\mathrm{CD} 271^{+}$cells compared with an unsorted population measured by Oil Red O and Alizarin Red stainings. The CD271 $1^{+}$population also possessed an increased proliferative rate compared with unsorted ASCs [12].

At last, an important in vivo study has suggested that CD271 $1^{+}$ASCs can be effectively transplanted. The authors depleted murine SVF of $\mathrm{Lin}^{+}$(lineage) ASCs, and the resulting Lin $^{-}$cells expressing both CD271 and Sca-1 (stem cell antigen-1) were selected. Lin and Sca-1 are typically used in this way as markers for hematopoietic stem cells, therefore the $\mathrm{Lin}^{-} / \mathrm{CD} 271^{+} / \mathrm{Sca}-1^{+}$population can be considered a purified stem cell population. Since most $\mathrm{CD} 271^{+}$cells are initially $\mathrm{Lin}^{-} / \mathrm{Sca}-1^{+}$, this population can be considered comparable to a pure CD271 ${ }^{+}$ population. This ASC population, when transplanted into mice in a fibrin construct, maintained the capacity to differentiate into adipocytes as measured by Oil Red $\mathrm{O}$ staining 6 weeks after implantation [21]. 


\section{Final thoughts}

The use of adipose tissue as a source of stem cell is a clinical reality that holds greater therapeutic potential than the current applications. As an autologous treatment, it avoids issues of rejection or requirement for immunosuppression. Surgeons are already very familiar with fat harvest techniques which are performed with low morbidity and there are several technologies in existence which seek to concentrate the harvested adipose tissue. There are also commercial technologies that digest fat to produce an SVF population within clinically appropriate time frames. The utility of subpopulation selection is yet to be demonstrated to be worthwhile clinically or economically, yet it appears to be the next step in providing precision treatments in personalized interventions.

Selecting ASC subpopulations based on cell surface marker proteins is feasible in both the laboratory and the clinic. It is therefore an attractive prospect for translation. The evidence described above demonstrates that they have distinct phenotypes as compared with the SVF and contain differential potencies for various applications in regenerative medicine. Although the basic characteristics of some ASC subpopulations have been elucidated, much knowledge is missing before translation to clinical practice can be achieved. It is likely that these ASC subpopulations work synergistically with others, and equally that there will be some subpopulations of cells within the SVF which dampen the potency of the intended intervention. Subsequently, subpopulations that can be shown to possess regenerative capacity in vitro, and retain this capacity in animal models, will be the prime candidates for translation to regenerative techniques in humans.

Subpopulations of ASCs, sorted from SVF with high levels of reproducibility, offer enhanced therapeutic value over heterogeneous populations due to a clearer understanding of their characteristics and potential applications. As regenerative medicine becomes more personalized, subpopulations of ASCs will be at the forefront of targeted therapies.

\section{Financial \& competing interests disclosure}

No financial support was received for the preparation of this manuscript. RJP Smith and AJ Reid are supported by the Scar Free Foundation, the National Institute for Health Research (II-LA-0313-20003), the Academy of Medical Sciences, the Hargreaves and Ball Trust and the Lumley Trust. The authors have no other relevant affiliations or financial involvement with any organization or entity with a financial interest in or financial conflict with the subject matter or materials discussed in the manuscript apart from those disclosed.

No writing assistance was utilized in the production of this manuscript.

\section{References}

1. Lockhart RA, Aronowitz JA, Dos-Anjos Vilaboa S. Use of freshly isolated human adipose stromal cells for clinical applications. Aesthet. Surg. J. 37(Suppl. 3), S4-S8 (2017).

2. Desiderio V, De Francesco F, Schiraldi C et al. Human Ng2+ adipose stem cells loaded in vivo on a new crosslinked hyaluronic acid-Lys scaffold fabricate a skeletal muscle tissue. J. Cell. Physiol. 228(8), 1762-1773 (2013).

3. Hiwatashi N, Hirano S, Mizuta M et al. Adipose-derived stem cells versus bone marrow-derived stem cells for vocal fold regeneration. Laryngoscope 124(12), E461-E469 (2014).

4. Block TJ, Garza JR. Regenerative cells for the management of osteoarthritis and joint disorders: a concise literature review. Aesthet. Surg. J. 37(Suppl. 3), S9-S15 (2017).

5. Kingham PJ, Kolar MK, Novikova LN, Novikov LN, Wiberg M. Stimulating the neurotrophic and angiogenic properties of human adipose-derived stem cells enhances nerve repair. Stem Cells Dev. 23(7), 741-754 (2014).

6. Zhu M, Zhou Z, Chen Y et al. Supplementation of fat grafts with adipose-derived regenerative cells improves long-term graft retention. Ann. Plast. Surg. 64(2), 222-228 (2010).

7. Ko MS, Jung JY, Shin IS et al. Effects of expanded human adipose tissue-derived mesenchymal stem cells on the viability of cryopreserved fat grafts in the nude mouse. Int. J. Med. Sci. 8(3), 231-238 (2011).

8. Kolle SF, Fischer-Nielsen A, Mathiasen AB et al. Enrichment of autologous fat grafts with ex vivo expanded adipose tissue-derived stem cells for graft survival: a randomised placebo-controlled trial. Lancet 382(9898), 1113-1120 (2013).

9. Gontijo-De-Amorim NF, Charles-De-Sa L, Rigotti G. Mechanical supplementation with the stromal vascular fraction yields improved volume retention in facial lipotransfer: a 1-year comparative study. Aesthet. Surg. J. 37(9), 975-985 (2017).

10. Baer PC. Adipose-derived mesenchymal stromal/stem cells: an update on their phenotype in vivo and in vitro. World J. Stem Cells 6(3), 256-265 (2014).

11. Rada T, Carvalho PP, Santos TC, Castro AG, Reis RL, Gomes ME. Chondrogenic potential of two hASCs subpopulations loaded onto gellan gum hydrogel evaluated in a nude mice model. Curr. Stem Cell Res. Ther. 8(5), 357-364 (2013). 
12. Quirici N, Scavullo C, De Girolamo L et al. Anti-L-NGFR and -CD34 monoclonal antibodies identify multipotent mesenchymal stem cells in human adipose tissue. Stem Cells Dev. 19(6), 915-925 (2010).

13. Miranville A, Heeschen C, Sengenes C, Curat CA, Busse R, Bouloumie A. Improvement of postnatal neovascularization by human adipose tissue-derived stem cells. Circulation 110(3), 349-355 (2004).

14. Chung MT, Liu C, Hyun JS et al. CD90 (Thy-1)-positive selection enhances osteogenic capacity of human adipose-derived stromal cells. Tissue Eng. Part A 19(7-8), 989-997 (2013).

15. De Francesco F, Tirino V, Desiderio V et al. Human CD34/CD90 ASCs are capable of growing as sphere clusters, producing high levels of VEGF and forming capillaries. PLoS ONE 4(8), e6537 (2009).

16. Ferraro GA, De Francesco F, Nicoletti G et al. Human adipose CD34+ CD90+ stem cells and collagen scaffold constructs grafted in vivo fabricate loose connective and adipose tissues. J. Cell. Biochem. 114(5), 1039-1049 (2013).

17. Son MY, Choi H, Han YM, Cho YS. Unveiling the critical role of REX1 in the regulation of human stem cell pluripotency. Stem Cells 31(11), 2374-2387 (2013).

18. Liu J, Sato C, Cerletti M, Wagers A. Notch signaling in the regulation of stem cell self-renewal and differentiation. Curr. Top. Dev. Biol. 92, 367-409 (2010).

19. Michalczyk K, Ziman M. Nestin structure and predicted function in cellular cytoskeletal organisation. Histol. Histopathol. 20(2), 665-671 (2005).

20. Yamamoto N, Akamatsu H, Hasegawa $S$ et al. Isolation of multipotent stem cells from mouse adipose tissue. J. Dermatol. Sci. 48(1), 43-52 (2007).

21. Xiao J, Yang X, Jing W et al. Adipogenic and osteogenic differentiation of Lin(-)CD271(+)Sca-1(+) adipose-derived stem cells. Mol. Cell. Biochem. 377(1-2), 107-119 (2013). 